\title{
Abscopal effect of unirradiated lung nodules following radiotherapy alone in recurrent non-small cell lung cancer: a case report
}

\author{
Ting-Chun Lin ${ }^{1 \#}$, Hsin-Yuan Fang ${ }^{2,3 \#}$, Ching-Yun Hsieh ${ }^{4 \#}$, Hsin-Cheng Hsu ${ }^{5 \#}$, Chia-Hung Kao ${ }^{3,6}$, \\ Su-Tso Yang ${ }^{7,8}$, Ji-An Liang ${ }^{1,3}$, Chun-Ru Chien ${ }^{1,3,9}$ \\ ${ }^{1}$ Department of Radiation Oncology, ${ }^{2}$ Department of Chest Surgery, China Medical University Hospital, Taichung, Taiwan; ${ }^{3}$ School of Medicine, \\ College of Medicine, China Medical University, Taichung, Taiwan; ${ }^{4}$ Division of Hematology and Oncology, Department of Internal Medicine, China \\ Medical University Hospital, Taichung, Taiwan; ${ }^{5}$ Department of Chinese Medicine, China Medical University Hsinchu Hospital, Hsinchu, Taiwan; \\ ${ }^{6}$ Department of Nuclear Medicine and PET Center, ${ }^{7}$ Department of Radiology, China Medical University Hospital, Taichung, Taiwan; ${ }^{8}$ School of \\ Chinese Medicine, China Medical University, Taichung, Taiwan; ${ }^{9}$ Department of Radiation Oncology, China Medical University Hsinchu Hospital, \\ Hsinchu, Taiwan \\ \#These authors contributed equally to this work. \\ Correspondence to: Chun-Ru Chien, MD, PhD. 2 Yuh-Der Road, Taichung, Taiwan. Email: d16181@mail.cmuh.org.tw.
}

\begin{abstract}
Abscopal effect is an intriguing phenomenon when tumor regression is observed at nonirradiated region distant from the primary irradiation site. Despite its popularity as a research topic (especially in combination with immunotherapy), seldom was the phenomenon observed when radiotherapy (RT) was the only treatment modality. Here we reported the case of a 67-year-old man with abscopal effect observed in the right lung lesions after RT to the recurrent left lung tumor. The patient was a case of metachronous early-stage squamous cell carcinoma (SqCC) of the head and neck and lung. He was otherwise healthy without any other underlying systemic disease, and had never received any medication or systemic treatment. The patient was diagnosed with early-stage left lower lobe SqCC and received postoperative adjuvant RT alone to the tumor bed. Bilateral lung tumor recurrence was observed after 6 months, and the patient completed RT alone to the left lung lesion. However, tumor regression was observed in the non-targeted right lung lesions. Interestingly, we noticed fluctuation in white blood cell (WBC) count around the course of palliative RT. We hope that this case can help secure physicians' attention to the systemic effect RT alone can contribute.
\end{abstract}

Keywords: Radiotherapy (RT); abscopal; non-targeted; non-small cell lung cancer (NSCLC); case report

Received: 02 January 2020; Accepted: 15 May 2020; Published: 30 September 2020.

doi: $10.21037 /$ tro-19-111

View this article at: http://dx.doi.org/10.21037/tro-19-111

\section{Introduction}

"Abscopal effect" has become a popular term in the academic field of oncology in this era of immunotherapy. However, long before the introduction of immunotherapy, the term "abscopal effect" was better known as "nontargeted radiotherapy (RT)", describing the occasionally observed phenomenon in which localized irradiation causes regression of another tumor at a distant site. Theories proposed generally include the direct cytotoxic effect on tumor cells which activates deoxyribonucleic acid (DNA) damage response pathways, and the impacts on the immune system which results in an immune-mediated anti-tumor response (1). Although the idea of inducing "non-targeted" tumor response sounds rather exciting, very few cases of abscopal effect have been reported where RT was the single treatment modality $(2,3)$. Most literature reported abscopal effect observed when RT was combined with other systemic treatment such as chemotherapy, target therapy or 
immunotherapy $(4,5)$. Melanoma and lymphoma were the most common histopathology, according to a systemic review of case reports on abscopal effect published in 2016 (3). Here we reported the case of a 67 -year-old man with non-small cell lung cancer (NSCLC) of the left lung with bilateral lung recurrence. Abscopal effect was observed in the right lung tumors after localized RT alone to the left lung tumor. We present the following case in accordance with the CARE reporting checklist (6).

\section{Case presentation}

This is a case of a 67-year-old man with metachronous cancer. Except for a history of occupational exposure to chemicals, the patient's lifestyle was otherwise healthy. $\mathrm{He}$ had smoked occasionally in the past but had quit for years, had no history of alcohol abuse, and had no family history of lung cancer. The patient was first diagnosed with earlystage right tongue squamous cell carcinoma ( $\mathrm{SqCC}$ ), clinical stage cT1N0M0, and received surgery alone in November 2015. The pathological stage was pT1N0M0. After 2 years of follow-up, he was diagnosed with a second cancer, a left lower lobe (LLL) NSCLC, in January 2017, for which he received sleeve lobectomy. The histopathology was SqCC, and surgical pathologic staging was $\mathrm{pT} 1 \mathrm{bN} 0, \mathrm{cM} 0$ with involved margin at the LLL bronchus. He further received postoperative adjuvant RT alone to the tumor bed for 60 Gray $(\mathrm{Gy}) / 30$ fractions (Fr) from February 2017 to March 2017. Following the treatment of the lung cancer, another early-stage left tongue SqCC was diagnosed and was treated with surgery alone in April 2017.

Unfortunately, the patient remained disease-free only for 6 months. In October 2017, a routine chest computed tomography (CT) revealed new bilateral lung metastases, which was further confirmed by positron emission tomography (PET) in November 2017. Bilateral lung lesions were detected. One larger lesion in LLL (maximal diameter $22 \mathrm{~mm}$; maximum standardized uptake value, SUVmax $=6.36$ ), and some smaller lesions in right lung (maximal joint diameter $8 \mathrm{~mm}$; SUVmax =3.23) (Figure 1). Physical examination revealed no abnormal findings, and biochemistry data was within normal limits. After multidisciplinary discussion for the optimal treatment strategy, the patient received RT alone with intensitymodulated radiation therapy (IMRT) technique for 60 Gy/20 Fr to the LLL lesion in January 2018 (RT course started on 2018/01/08, ended on 2018/02/02) (Figure 1). Throughout the disease course, RT was the only treatment modality involved and was well-tolerated without obvious side effect. The patient denied applying herbal medicine or any other alternative treatment. Interestingly, soon after he completed the palliative RT treatment course, we noticed a sudden drop of white blood cell (WBC) count $(4,100 / \mu \mathrm{L}$ on 2018/02/02) followed by a temporary elevation of WBC count $(8,800 / \mu \mathrm{L}$ on $2018 / 02 / 09)$ deviated from his baseline data (around $6,400 / \mu \mathrm{L}$ ), and then dropped back to baseline level within 3 months after RT completion (Figure 2). There was no sign of radiation pneumonitis and the patient had no complaint of cough or dyspnea.

Follow-up PET scan in February 2018 (Figure 3, $2^{\text {nd }}$ column) and July 2018 (Figure 3, $3^{\text {rd }}$ column) showed faint fluorodeoxyglucose (FDG) uptake in the right lung (highest SUVmax $=1.04$ ) and left pleural inflammation. Chest CT showed that the unirradiated right lung lesions shrank in November 2018 (Figure 4, left), and the chest CT done in May 2019 even showed further resolution of the right lung lesions (Figure 4, right). Follow-up PET/CT in January 2019 also showed complete metabolic response of LLL cancer and no F-18 FDG-avid distant metastasis (Figure 3, $4^{\text {th }}$ column). Another PET/CT done in September 2019 (Figure $3,5^{\text {th }}$ column) showed no apparent change compared with the last scan.

The patient's triple cancer remained progression free with continued presence of minimal disease in bilateral lungs as shown in his timeline (Figure 5). His clinical condition showed stability with good performance status (Eastern Cooperative Oncology Group Performance Status =1) with his latest follow-up visit to our clinic in December 2019 , and had never required any systemic therapy. Currently, the patient is under regular outpatient clinic follow-up every 3 months.

\section{Discussion}

Although the abscopal effect has been reported in various types of malignancies, it is a rarely recognized and widely debatable clinical event. In this case report, we observed persistent tumor regression in the non-targeted right lung lesions following palliative RT to an LLL lesion. There was no low dose area in the right lung lesions, and thus direct or bystander effect of radiation dose cannot explain tumor regression in this reported case. A pitfall of this report is lack of pathological proof for the recurrent bilateral lung lesions. Although metastases were the only impression in the formal report of CT in October 2017 and PET in November 2017, we cannot exclude other possibilities such as inflammation process or infection. Nonetheless, 


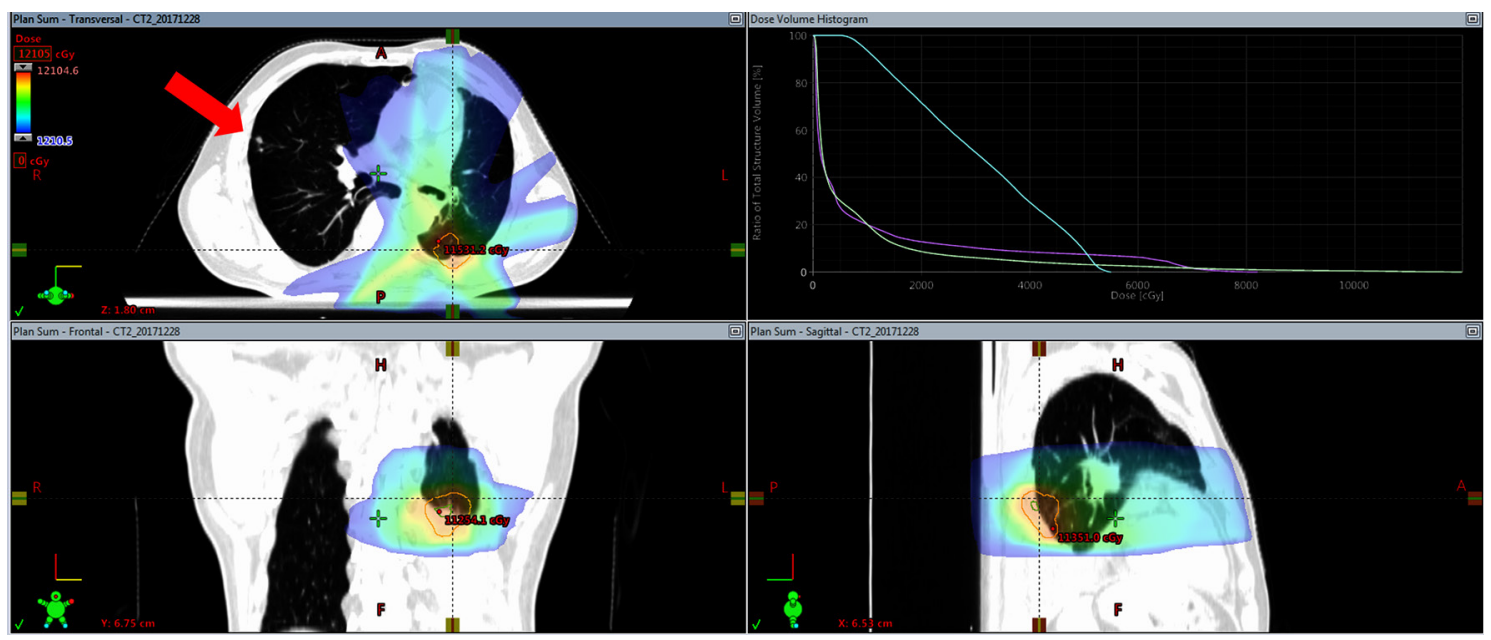

Figure 1 Dose-color wash image of the summed RT treatment plan for the left lung lesion. The non-targeted lesion is annotated with a red arrow. RT, radiotherapy.

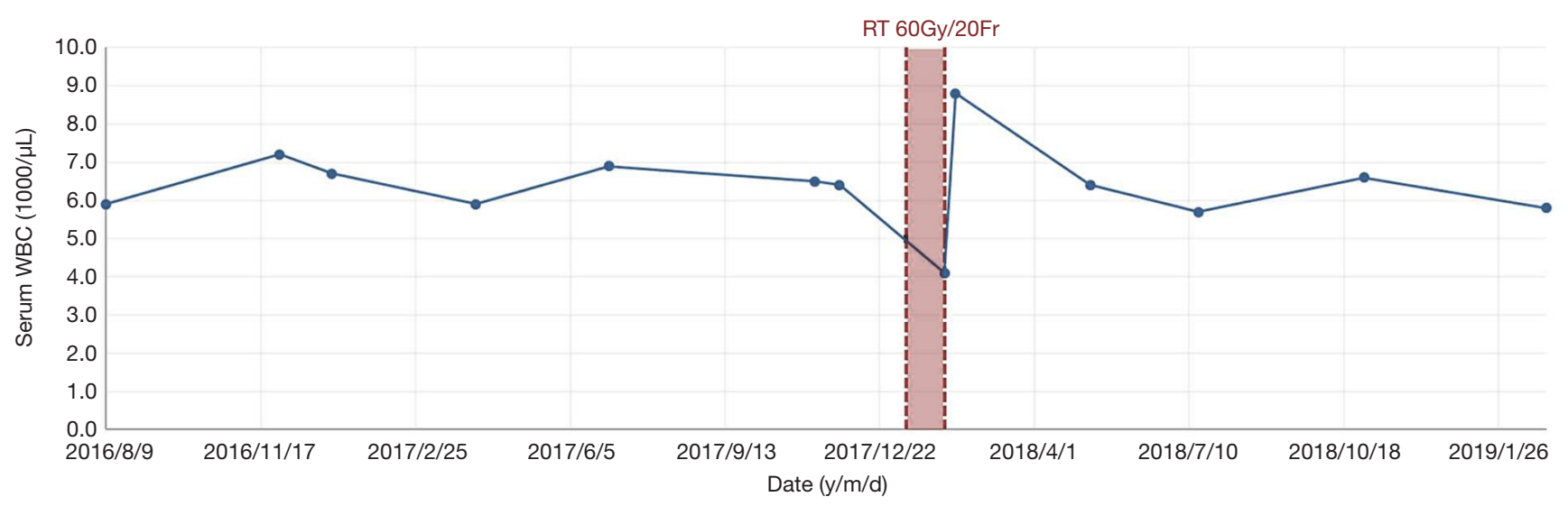

\begin{tabular}{|c|c|c|c|c|c|c|c|c|c|c|c|c|}
\hline $2016 / 8 / 9$ & $016 / 11 / 29$ & $2017 / 1 / 2$ & $2017 / 4 / 5$ & $2017 / 6 / 30$ & $2017 / 11 / 10$ & $2017 / 11 / 26$ & \begin{tabular}{|l|}
$2018 / 2 / 2$ \\
\end{tabular} & $2018 / 2 / 9$ & $2018 / 5 / 7$ & 2018/7/16 & 2018/10/31 & $2019 / 2 / 26$ \\
\hline 5.9 & 7.2 & 6.7 & 5.9 & 6.9 & 6.5 & 6.4 & 4.1 & 8.8 & 6.4 & 5.7 & 6.6 & 5.8 \\
\hline
\end{tabular}

Figure $2 \mathrm{WBC}$ data at baseline level before RT, throughout the RT course, and during follow-up. RT to the left lung lesion for 60 Gy/20 Fr was delivered from 2018/01/08 to 2018/02/02. RT, radiotherapy; WBC, white blood pressure.

as abscopal effect is usually observed in the setting of metastatic or recurrent disease, tissue biopsy or surgery is seldom arranged, and patients are usually treated under clinical diagnoses alone.

The sudden increase in WBC count at the end of the RT course in this patient is also worth attention. While most older literature stated that RT is immunosuppressive, a temporary leukopenia and then elevated WBC count was observed during the course of RT (7). Similar increase in $\mathrm{WBC}$ had also been reported in the literature (8). In patients treated with lung RT, the most common adverse event associated with elevated WBC count is radiation pneumonitis, which usually occurs within 6 months after RT, with a peak onset at 1 to 3 months $(9,10)$. As the patient reported no dyspnea and the WBC count soon returned to baseline level, diagnosis of radiation pneumonitis is unlikely. We presume that the localized irradiation somehow activated the immune system of this patient through a mechanism yet to be elucidated. In any case, the association between the activation of immune system and RT as a single treatment modality cannot be neglected in this report. However, further studies are required to clarify 

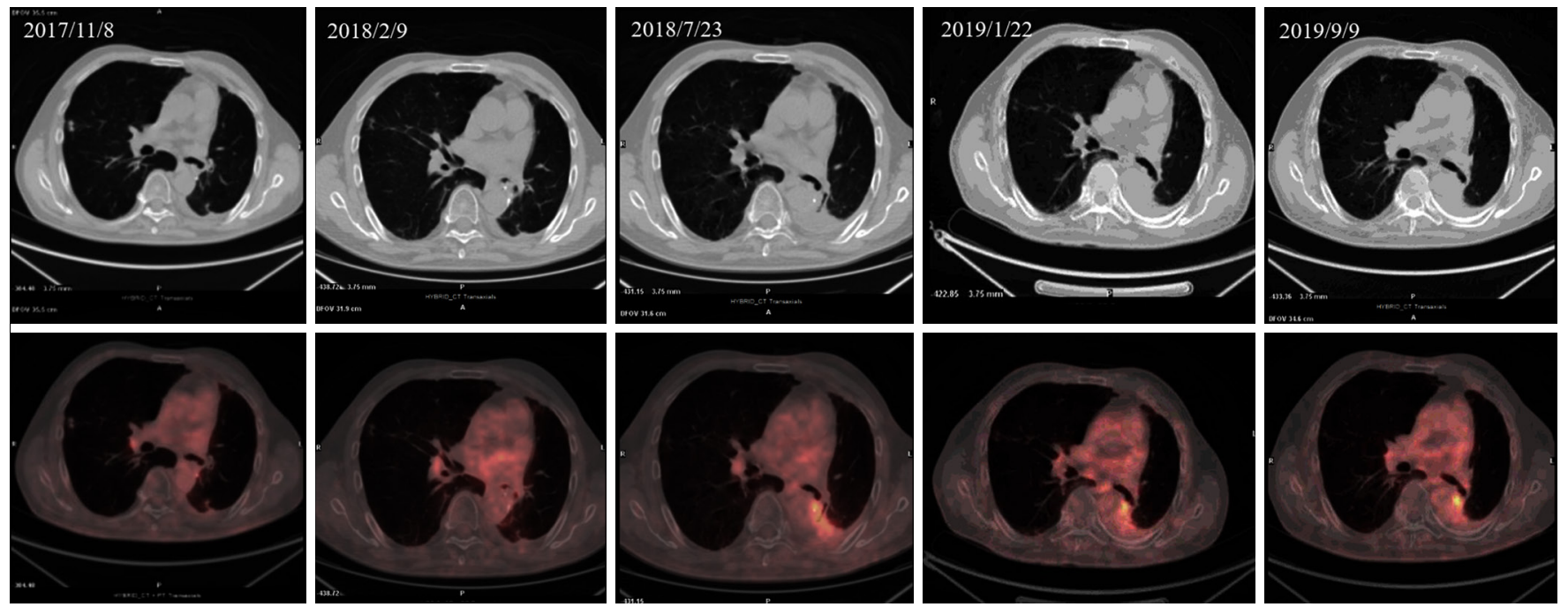

Figure 3 The first column to the fifth column (left to right): baseline PET/CT scan done on November 8th, 2017 (1st column). Followup PET/CT scan was done on February 9th, 2018 (2nd column), July 23rd, 2018 (3rd column), January 22nd, 2019 (4th column), and September 9th, 2019 (5th column).
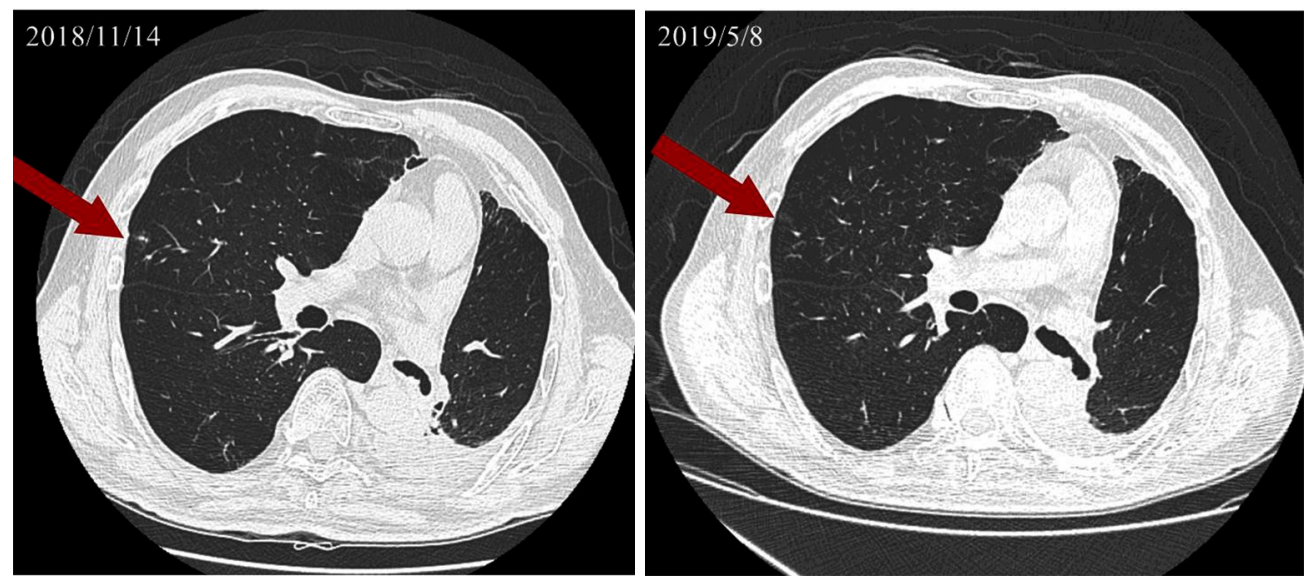

Figure 4 Follow-up chest CT scan done on November $14^{\text {th }}$, 2018 showed regression in right lung lesion (left). Chest CT on May $8^{\text {th }}$, 2019 showed complete remission of the right lung lesion (right). The lesion is marked with red arrows. CT, computed tomography.

this observation.

Over the past few years, rapidly growing preclinical and clinical studies have showed that the combination of local RT and immunotherapy induces anti-tumor immunity synergistically, thus enhancing abscopal effect $(4,5,11)$. Recent laboratory and preclinical studies have also demonstrated that immune system can be activated by local RT alone, although the mechanism remains elusive. The potential role that RT may have in systemic cancer treatment has never attracted so much attention. However, blood and biochemistry data were seldom mentioned in published case reports of abscopal effect, implying that even radiation oncologists tend to ignore the systemic effect RT alone can induce. We hope that in this era of precision medicine and integrated treatment, this case report could bring radiation oncologists' attention to this fascinating observation and help physicians embrace more possibilities in future cancer treatment.

\section{Conclusions}

This case report demonstrates the rare clinical phenomenon 


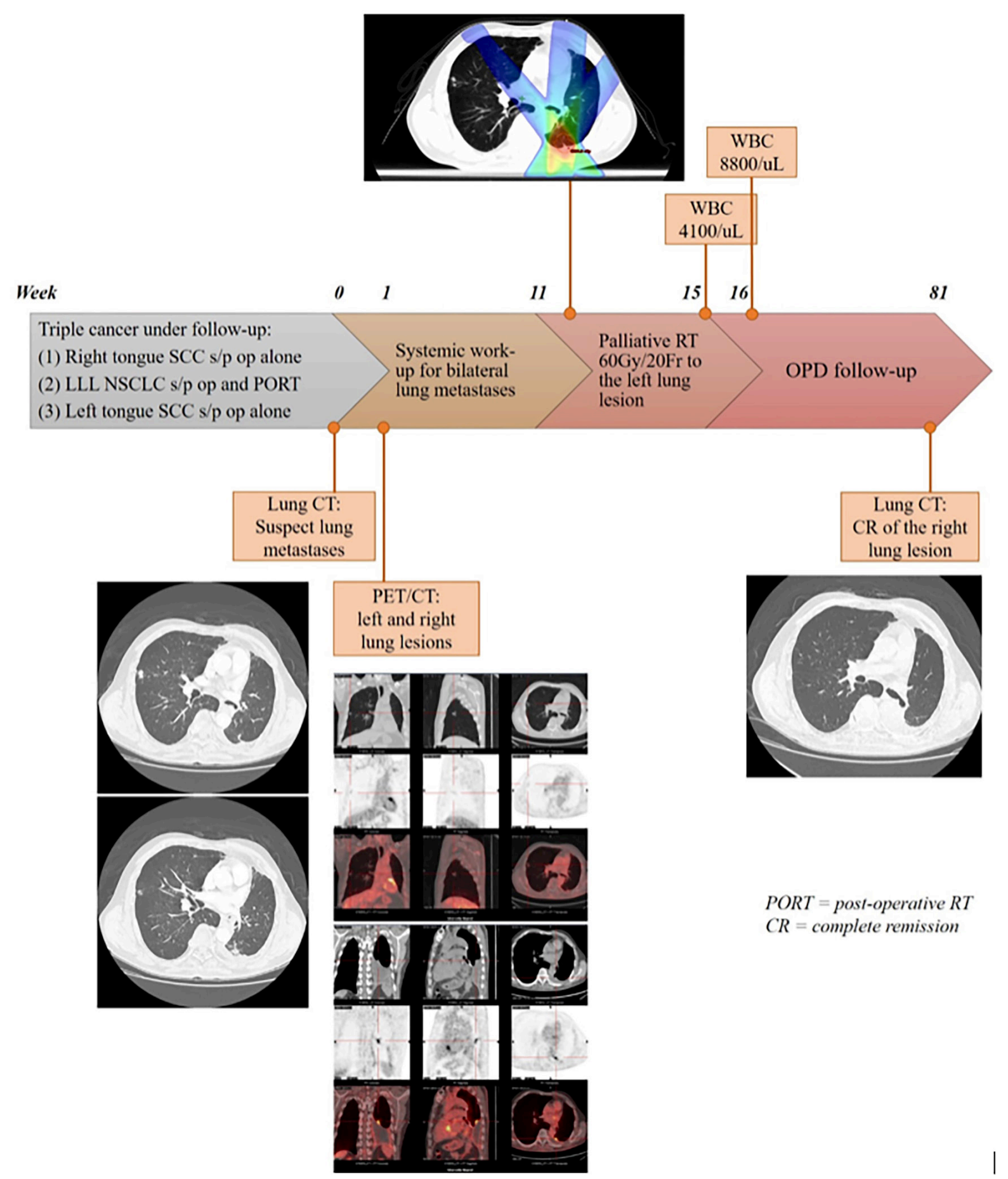

Figure 5 Timeline of this patient. WBC, white blood cell; LLL, left lower lobe; NSCLC, non-small cell lung cancer; SCC, squamous cell carcinoma; RT, radiotherapy; PORT, post-operative RT; OPD, outpatient department; PET, positron emission tomography; CT, computed tomography. 
that abscopal effect could be induced by RT alone in a case of NSCLC with bilateral lung recurrence. Moreover, it provides clinical evidence that RT as a single treatment modality may be capable of inducing immunomodulation effect without systemic intervention.

\section{Acknowledgments}

This study has been presented in part at the $23^{\text {th }}$ Taiwan Joint Cancer Conference in Taipei, Taiwan, R.O.C. on May $5^{\text {th }}$ and $6^{\text {th }}$ in 2018 .

Funding: None.

\section{Footnote}

Provenance and Peer Review: This article was commissioned by the Guest Editors (Chen-Hsi Hsieh, Hsin-Hua Nien, and Yu-Ming Huang) for the series "Precision Medicine for Cancer" published in Therapeutic Radiology and Oncology. The article has undergone external peer review.

Conflicts of Interest: All authors have completed the ICMJE uniform disclosure form (available at http://dx.doi. org/10.21037/tro-19-111). The series "Precision Medicine for Cancer" was commissioned by the editorial office without any funding or sponsorship. JAL serves as an unpaid editorial board member of Therapeutic Radiology and Oncology from Apr 2020 to Mar 2022. The authors have no other conflicts of interest to declare.

Ethical Statement: The authors are accountable for all aspects of the work in ensuring that questions related to the accuracy or integrity of any part of the work are appropriately investigated and resolved. All procedures performed in studies involving human participants were in accordance with the Declaration of Helsinki (as revised in 2013). The study is approved by the Institutional Review Board (IRB) of China Medical University Hospital in Taichung, Taiwan [CMUH106-REC3-119 (CR2)]. The patient is happy with the treatment result and written informed consent was obtained from the patient for publication of this case report and any accompanying images. Patient specific information was de-identified.

Open Access Statement: This is an Open Access article distributed in accordance with the Creative Commons Attribution-NonCommercial-NoDerivs 4.0 International License (CC BY-NC-ND 4.0), which permits the non- commercial replication and distribution of the article with the strict proviso that no changes or edits are made and the original work is properly cited (including links to both the formal publication through the relevant DOI and the license). See: https://creativecommons.org/licenses/by-nc-nd/4.0/.

\section{References}

1. Yilmaz MT, Elmali A, Yazici G. Abscopal Effect, From Myth to Reality: From Radiation Oncologists' Perspective. Cureus 2019;11:e3860.

2. Bitran J. The Abscopal Effect Exists in Non-small Cell Lung Cancer: A Case Report and Review of the Literature. Cureus 2019;11:e4118.

3. Abuodeh Y, Venkat P, Kim S. Systematic review of case reports on the abscopal effect. Curr Probl Cancer 2016;40:25-37.

4. Hlavata Z, Solinas C, De Silva P, et al. The Abscopal Effect in the Era of Cancer Immunotherapy: a Spontaneous Synergism Boosting Anti-tumor Immunity?. Target Oncol 2018;13:113-23.

5. Reynders K, Illidge T, Siva S, et al. The abscopal effect of local radiotherapy: using immunotherapy to make a rare event clinically relevant. Cancer Treat Rev 2015;41:503-10.

6. Riley DS, Barber MS, Kienle GS, et al. CARE guidelines for case reports: explanation and elaboration document. J Clin Epidemiol 2017;89:218-35.

7. Wara WM. Immunosuppression associated with radiation therapy. Int J Radiat Oncol Biol Phys 1977;2:593-6.

8. Mikuriya S, Kamimura S, Kato T, et al. Relationship Between Radiotherapy and Tumor Immunity in Hosts. Gan No Rinsho 1983;29:1521-36.

9. Jain V, Berman AT. Radiation Pneumonitis: Old Problem, New Tricks. Cancers (Basel) 2018;10:222.

10. Wall RJ, Schnapp LM. Radiation pneumonitis. Respir Care 2006;51:1255-60.

11. Rodríguez-Ruiz ME, Vanpouille-Box C, Melero I, et al. Immunological Mechanisms Responsible for RadiationInduced Abscopal Effect. Trends Immunol 2018;39:644-55.

doi: $10.21037 /$ tro-19-111

Cite this article as: Lin TC, Fang HY, Hsieh CY, Hsu HC, Kao CH, Yang ST, Liang JA, Chien CR. Abscopal effect of unirradiated lung nodules following radiotherapy alone in recurrent non-small cell lung cancer: a case report. Ther Radiol Oncol 2020;4:19. 